\title{
测绘工程中无人机技术的应用探讨
}

\author{
蒋德洪 \\ 山东金桥建设项目管理有限公司，山东滩坊 262700
}

[摘要]近年来，在多方面利好因素的影响下我国科学技术得到了良好的发展进步，为无人机技术水平的提升起到了积极的辅 助作用。无人机技术自身拥有良好的实用性，所以受到了人们的广泛青睐，被大范围的运用到了相关领域之中，并且取得了 良好的成绩。在将无人机技术加以实践运用的时候, 可以借助无人机搭载专业遥感设备来获取需要的影像资料, 收集相关数 据信息, 这样就可以在不同的环境下完成测量测绘工作, 从而对以往老旧测绘技术中存在的问题加以解决。其次, 在建筑工 程行业飞速发展的形势下，无人机技术得以大范围的运用，对于相关专业领域的发展是非常有帮助的。

[关键词]测绘; 工程; 无人机技术

DOI: $10.33142 /$ sca.v4i3.4024 中图分类号: P23 文献标识码: A

\section{Application of UAV Technology in Surveying and Mapping Engineering}

\author{
JIANG Dehong
}

Shandong Jinqiao Construction Project Management Co., Ltd., Weifang, Shandong, 262700, China

\begin{abstract}
In recent years, under the influence of many favorable factors, Chinese science and technology has made good progress, which has played a positive auxiliary role in the improvement of UAV technology level. UAV technology has good practicability, so it is widely favored by people, has been widely used in related fields and has achieved good results. In the practical application of UAV technology, we can use UAV equipped with professional remote sensing equipment to obtain the required image data and collect relevant data information, so that we can complete the surveying and mapping work in different environments, so as to solve the problems existing in the old surveying and mapping technology. Secondly, with the rapid development of the construction industry, UAV technology can be widely used, which is very helpful for the development of related professional fields.
\end{abstract}

Keywords: surveying and mapping; engineering; UAV technology

在科学技术飞速发展的带动下, 大量的新型科学技术被人们研发出来, 其中无人机遥感技术是当前最为先进的一 种科学技术, 将其运用到测绘工程之中对于提升测绘工作的效率和质量能够起到积极的辅助作用。

\section{1 无人机测绘技术}

1.1 无人机测绘系统组成

无人机测绘系统通常都是由硬件和软件两个部分组合而成, 其中硬件涉及到无人机飞行器、无人机桨翼及高清摄 像机等, 软件往往都是由无人机控制系统、飞行器操作、软件系统、遥感系统、无线电遥控系统、图像处理软件等几 部分组成。无人机的材质一般都是运用的合金、碳纤维、玻璃钢的材料制造而成, 在机身结构中安设电池、降落伞仓 等分支结构, 并且会在机身结构上安放专门的专业高清摄像设备。结合无人机桨翼的实际模式, 无人机可以分为固定 翼和多旋翼无人机两种不同的形式。

\section{2 无人机测绘工作原理}

无人机的测绘系统可以划分为传感器、机载计算机、伺服驱动系统几个不同的系统, 在正式开始测量工作之前, 应当充分结合各方面实际情况来挑选适合的无人机, 之后结合实际情况和需要来设计无人机飞行航线, 从而保证能够 全面的获取需要的信息数据 ${ }^{[1]}$ 。

\section{2 无人机技术在测绘工程中的应用优势}

\section{1 测绘效率较高}

与之前老旧模式的测绘方法相对比来看, 无人机技术的实践运用范围更加的宽泛, 实用性相对较强, 施工单位在 组织实施工程测绘工作的时候, 如果遇到任何的问题, 可以运用专业的技术加以解决, 尽可能的提升测绘工作的效率 和质量, 切实的规避经济损失情况的发生。无人机遥感技术的运用可以能够较为高效的对各类突发问题加以解决, 在 
测绘工作中发挥出重要的辅助作用, 切实的规避各类风险情况的发生 ${ }^{[2]}$ 。

\section{2 信息处理迅速}

工作人员在实际运用无人机进行目标对象测绘工作的时候, 能够更加高效的确定测绘目标, 保证测绘结果的准确 性。就以往测绘工作来说, 往往会遇到测绘范围界定不清楚的情况, 所以会对测绘工作的效果造成一定的损害。无人 机安设了专门的高分辨率摄像设备, 可以实现全面的收集信息的目的, 从而为后续各项工作的实施给予良好的辅助。

\section{3 监测尺度较大}

在科学技术快速发展的推动下, 无人机技术的水平得到了快速的提升, 带动了相关硬件设备和软件系统的优化和 完善, 专业技术人员可以借助高水平的无人机技术来对目标进行检测, 结合测绘工作实际情况和需要来对监测尺度进 行适当的调整, 这样不但可以切实的对测量范围加以把控, 并且也具备良好的伸缩性。其次, 无人机技术能够将整个 测量区域的实际情况, 运用三维成像技术来呈现出来, 为各项工作的实施给予一定的参考 ${ }^{[3]}$ 。

\section{3 无人机技术的发展现状}

无人机技术其实质就是运用专业的无线设备来对飞行器来进行操控, 这样就可以掌握充足的信息数据。无人机技 术是当前最为先进的一种科学技术, 将其加以实践运用能够有效的解决以往老旧地面测绘工作中所存在的诸多问题, 提升测绘工作的效率和质量, 保证测绘结果的准确性, 因为自身具有良好的优越性所以受到了人们的广泛青睐, 被大 范围的运用到了诸多领域之中。

\section{4 无人机技术的应用}

\section{1 测量范围拓展}

如果需要测绘的范围较为广泛, 那么单纯的运用人工测绘的方法, 会为测绘工作人员带来巨大的工作量, 这样必 然会对测绘工作的效率和质量的提升造成一定的限制。并且人工测绘的效果往往会受到测绘工作人员的专业水平和综 合素质的影响, 无法从根本上对测绘结果的准确性加以保证。而将无人机技术切实的运用到测绘工作之中, 不但可以 有效的促进测绘范围的扩展, 并且也能够更加高效的对测绘目标周边物体情况加以掌握, 对于提升测绘工作的质量是 非常有效的。无人机具备良好的分辨率, 所以在进行测绘工作的时候, 拍摄的质量和效果得到了根本保障。将 4RTK 航 拍器加以实践运用, 能够实现对测绘区域的准确测绘, 不但可以对测绘获得的结果的准确性加以保证, 并且也可以完 成对相关内容的矫正和检查, 这样就可以避免测绘结果错误的情况发生。其次, 在实际组织开展测绘工作的时候, 测 绘位置涉及到很多的区域往往是人工测绘无法实现的, 而借助无人机技术能够切实高效的对测绘数据进行收集, 并且 将当前最先进的影像分析技术加以实践运用, 综合现如今 $3 \mathrm{D}$ 建模软件能够有效的提升测量工作的整体水平, 将测绘标 的物准确的呈现出来 ${ }^{[4]}$ 。

\section{2 地形测量效率提升}

地形测量工作能够更加准确的将地面地势情况以及地势的特征加以全面的掌握, 为后续相关区域的建设工作给予 良好的帮助。诸如: 某个项目测绘工作持续一百四十分钟, 利用两台无人机进行测绘。如果工作人员在没有实施地面 勘察工作的情况下来实施测绘工作, 并且测绘内容的分辨力为 $9 \mathrm{~cm}$, 这样就可以其实的满足测绘工作的实际需要, 这样 对于地形测量工作整体水平的提升是非常有帮助的。与人工测绘工程量相对比来看, 无人机测绘在提升测绘效率方面 能够起到良好的作用, 并且环保性能较强, 不需要进行前期调研, 结合现如今最为先进的无人机地面测绘技术来进行 实践测绘工作, 因为测绘范围内的地面建筑较多, 对于测绘区域会产生诸多的干扰, 但是运用专业的图像计算软件能 够保证地面数据的准确的测算, 这就充分的说明了, 地形测绘效率的不断提升, 能够为后续各项工作的高效开展起到 良好的协助作用。

\section{3 测绘成本下降}

无人机的测绘效率在多方面利好因素的影响下得到了显著的提升, 从而为后期各项工作的实施打下了良好的基础, 也可以有效的控制测绘工作的成本。针对测量设备以及后期图像成像软件等相关费用综合分析我们发现, 无人机的测 量费用不会超出人工测量的费用, 并且测量结果的准确性更高。所以能够有效的缩减整个工程的成本。但是不得不说 的是, 借助无人机技术来实施测绘工作的时候, 可以借助租赁模式来推进各项工作, 这样对于提升测绘工作的效率和 质量都是非常有帮助的。为了保证测绘的规范性, 应当结合无人机的测绘实际需要来挑选适合的无人机设备 ${ }^{[5]}$ 。无人机 技术拥有较强的价值, 合理的运用无人机技术可以切实的控制工程成本, 但是在将这项技术加以实践运用的时候对于 
环境因素提出了更高的要求, 特别是遇到降雨或者是雷雨天气的时候不适合运用无人机设备来进行测绘工作。

\section{4 联动优势}

在测绘工程汇总将无人机技术加以实践运用, 并且利用电子设备来进行信息收据的统一收集和处理, 确保信息数 据的准确性, 并且针对测量内容加以综合分析, 利用专业的软件系统创设立体模型, 从而将测绘工作的作用彻底的发 挥出来。

\section{5 结论}

总的来说，借助无人机技术来实施工程测绘工作不但可以提升测绘结果的准确性，并且也可以为后续工作实施给 予帮助, 控制测绘成本, 提升整个工程的整体经济性, 创设多维度动态测绘模型, 为后续工程各项建设工作的实施起 到良好帮助。

\section{[参考文献]}

[1]陈剑峰.测绘工程中无人机技术的应用探讨 [J].中国住宅设施, 2020(12): 52-53.

[2] 聂建伟. 研究无人机技术在测绘工程中的应用 [J]. 工程建设与设计,2020(22) : 253-254.

[3] 于庆启.测绘工程中无人机技术的应用研究 [J]. 建材与装饰, 2020(7) : 252-253.

[4] 梁艳. 试析测绘工程中无人机技术的应用价值及实践应用策略 [J]. 城市建设理论研究 (电子版), 2019 (4): 96.

[5]张涵.无人机在测绘工程中应用技术的分析 [J]. 硅谷, 2014,7 (16): 127-128.

作者简介: 蒋德洪 (1968.1-), 毕业院校: 国家开放大学, 所学专业: 土木工程, 当前就职单位: 山东金桥建设项目 管理有限公司，职务：副总，职称级别：副高级工程师。 